\title{
Multi-patient study for coronary vulnerable plaque model comparisons: 2D/3D and fluid-structure interaction simulations
}

\author{
Qingyu Wang ${ }^{1} \cdot$ Dalin Tang $^{1,2}\left(\mathbb{D} \cdot\right.$ Liang Wang $^{1} \cdot$ Akiko Meahara $^{3} \cdot$ David Molony $^{4} \cdot$ Habib Samady $^{4} \cdot$ Jie Zheng $^{5}$. \\ Gary S. Mintz ${ }^{3}$. Gregg W. Stone ${ }^{3,6}$. Don P. Giddens ${ }^{4,7}$
}

Received: 20 December 2020 / Accepted: 7 March 2021 / Published online: 23 March 2021

(c) The Author(s) 2021

\begin{abstract}
Several image-based computational models have been used to perform mechanical analysis for atherosclerotic plaque progression and vulnerability investigations. However, differences of computational predictions from those models have not been quantified at multi-patient level. In vivo intravascular ultrasound (IVUS) coronary plaque data were acquired from seven patients. Seven 2D/3D models with/without circumferential shrink, cyclic bending and fluid-structure interactions (FSI) were constructed for the seven patients to perform model comparisons and quantify impact of 2D simplification, circumferential shrink, FSI and cyclic bending plaque wall stress/strain (PWS/PWSn) and flow shear stress (FSS) calculations. PWS/PWSn and FSS averages from seven patients (388 slices for 2D and 3D thin-layer models) were used for comparison. Compared to 2D models with shrink process, 2D models without shrink process overestimated PWS by $17.26 \%$. PWS change at location with greatest curvature change from 3D FSI models with/without cyclic bending varied from $15.07 \%$ to $49.52 \%$ for the seven patients (average $=30.13 \%$ ). Mean Max-FSS, Min-FSS and Ave-FSS from the flow-only models under maximum pressure condition were $4.02 \%, 11.29 \%$ and $5.45 \%$ higher than those from full FSI models with cycle bending, respectively. Mean PWS and PWSn differences between FSI and structure-only models were only $4.38 \%$ and $1.78 \%$. Model differences had noticeable patient variations. FSI and flow-only model differences were greater for minimum FSS predictions, notable since low FSS is known to be related to plaque progression. Structure-only models could provide PWS/PWSn calculations as good approximations to FSI models for simplicity and time savings in calculation.
\end{abstract}

Keywords Vulnerable plaque $\cdot$ VH-IVUS $\cdot$ Patient-specific model $\cdot$ Models comparison

Dalin Tang

dtang@wpi.edu

1 School of Biological Science and Medical Engineering, Southeast University, Nanjing 210096, China

2 Mathematical Sciences Department, Worcester Polytechnic Institute, 100 Institute Road, Worcester, MA 01609, USA

3 The Cardiovascular Research Foundation, Columbia University, New York, NY 10022, USA

4 Department of Medicine, Emory University School of Medicine, Atlanta, GA 30307, USA

5 Mallinckrodt Institute of Radiology, Washington University, St. Louis, MO 63110, USA

6 Icahn School of Medicine At Mount Sinai, The Zena and Michael A. Wiener Cardiovascular Institute, New York, NY 10019, USA

7 Department of Biomedical Engineering, The Wallace H. Coulter, Georgia Institute of Technology, Atlanta, GA 30332, USA

\section{Introduction}

Computational models are powerful tools that have been used to perform mechanical analysis on atherosclerotic plaques and identify risk factors that may be related to plaque progression and rupture (Cardoso et al. 2014; Friedman et al. 2010; Gijsen et al. 2014; Holzapfel et al. 2014; Tang et al. 2014). Results from different models are influenced by many factors including plaque morphology and components, material properties, and modeling assumptions (Tang et al. 2014). With advances of medical imaging technologies, it is possible to obtain plaque morphology in vivo (Mintz et al. 2001; Nair et al. 2002). Two-dimensional (2D) image-based plaque solid models have previously been used to calculate stress/strain conditions of atherosclerotic plaques and to study their relationship to plaque progression and rupture (Li et al. 2006; Loree et al. 1992; Richardson et al. 1989). Considering the three-dimensional (3D) 
geometric structure of plaque, 3D structure-only models, 3D fluid-only models and 3D fluid-structure interaction (FSI) model have been used for the mechanical analysis of 3D plaque structure (Bluestein et al. 2008; Holzapfel et al. 2002; Ohayon et al. 2005; Samady et al. 2011; Stone et al. 2012; Tang et al. 2004; Teng et al. 2010). However, because of the complex geometry and composition of plaque, the construction of $3 \mathrm{D}$ plaque models is time-consuming. Various modeling strategies, including 2D structure-only model, 3D structure-only model and 3D FSI model, have been compared to investigate the differences in stress and strain analysis (Huang et al. 2014; Tang et al. 2004; Wang $\mathrm{H}$ et al. 2015). To improve the 2D model, a 3D thin-layer (TL) modeling method was used to replace 3D FSI model (Guo et al. 2018; Huang et al. 2016). However, previously published comparison of models mostly analyzed a single patient or models with idealized geometries. Multi-patient studies are more likely to demonstrate differences between models and according to patient variations.

For models based on in vivo data, it is important to obtain the initial zero-stress geometry of the plaque (Delfino et al. 1997; Holzapel et al. 2007; Huang et al. 2009; Ohayon et al. 2007; Pierce et al. 2015; Speelman et al. 2009; Wang L et al. 2017). Obtaining vessel's initial zero-stress state from its in vivo stressed geometric state requires quantifying the artery opening angle, axial and circumferential shrinkages (Wang L. et al. 2017). Circumferential shrinkage is defined as the circumferential (radial) contraction ratio that causes the vessel to change from in vivo geometry to no-load geometry in circumferential direction. Circumferential shrinkage may be quantified from vessel deformation under pulsating pressure conditions. A shrink-stretch process was proposed to obtain vessel no-load morphology (ignoring opening angle) from its in vivo image data (Huang et al. 2009). Quantifying artery opening angle and axial stretch ratio for in vivo data is not possible due to absent tissue samples. Existing ex vivo data from available literature are often used for model constructions as an acceptable simplification.
For coronary plaques, ventricle contraction and motion cause vessel curvature change which has considerable impact on plaque biomechanical conditions. Yang et al. introduced 3D FSI model with cyclic bending to include cardiac motion for mechanical analysis of coronary plaques (Yang et al. 2009). Due to patient variations, it is important to perform multi-patient studies to evaluate the effect of cyclic bending on plaque mechanical behaviors.

In the present study, in vivo VH-IVUS images and X-ray angiographic data of coronary plaques from seven patients were obtained for model construction. Seven different models were constructed for all seven patients for comparisons. Our goals were to quantify: (1) the influence of circumferential shrink process on 2D model calculation in different patients; (2) the impact of cyclic bending process on 3D FSI model in different patients; (3) the differences between 2D model, 3D TL model and 3D FSI model in different patients; (4) the differences between 3D structure-only model and 3D FSI model in different patients; (5) the differences between 3D fluid-only model and 3D FSI model in different patients. Patient variations for those model differences were also quantified.

\section{Data, models and methods}

\subsection{In vivo IVUS data acquisition}

IVUS with radiofrequency "virtual" histology (VH-IVUS) data of coronary plaque data were acquired from seven patients (mean age: 62, 6 males) using a synthetic-aperturearray, 20-MHz, 3.2-French catheter (Eagle Eye, Volcano Corporation, Rancho Cordova, California) at the Cardiovascular Research Foundation (CRF) with informed consent obtained. Morphological information of seven patients is shown in Table 1. Locations of the coronary artery stenosis and vessel curvature were obtained from X-ray angiogram data. The centerline of each frame of X-ray angiography film

Table 1 Patient information

\begin{tabular}{llllllll}
\hline Patient & Age & Gender & BP $(\mathrm{mmHg})$ & L-vessel $(\mathrm{mm})$ & $\begin{array}{l}\text { Number of } \\
\text { slices }\end{array}$ & Stenosis $(\%)$ & PB (\%) \\
\hline P1 & 71.3 & Male & $70-125$ & 70.58 & 59 & 45.97 & 73.52 \\
P2 & 72.3 & Male & $70-125$ & 70.00 & 61 & 61.87 & 82.51 \\
P3 & 67.5 & Male & $70-120$ & 28.96 & 56 & 5.79 & 58.23 \\
P4 & 51.6 & Male & $60-135$ & 33.92 & 64 & 38.73 & 68.45 \\
P5 & 51 & Male & $97-144$ & 35.37 & 40 & 35.59 & 69.08 \\
P6 & 67 & Female & $70-110$ & 55.02 & 44 & 56.57 & 78.10 \\
P7 & 52.1 & Male & $60-135$ & 33.98 & 64 & 43.26 & 73.61 \\
\hline
\end{tabular}

L-Vessel: vessel segment length

Stenosis $=(1-($ min lumen area/inlet lumen area $)) \times 100 \%$

$P B$ Plaque burden $=[($ wall area - lumen area $) /$ wall area $] \times 100 \%$ 
was extracted to describe the vessel curvature. The maximum vessel curvature and the minimum vessel curvature were selected from a cardiac cycle to simulate the cardiac cyclic bending of the coronary. Figure 1 shows selected VHIVUS images, their segmented contours, the $\mathrm{X}$-ray angiography image, and the re-constructed 3D geometry of the vessel.

\subsection{List of models, governing equations, boundary conditions and material models}

\subsubsection{Model list, governing equations and boundary conditions}

Various computational models have been used to perform flow shear stress and plaque stress and strain calculations, seeking their associations with plaque progression and rupture. Table 2 lists seven models considered in this paper. The assumptions, governing equations and boundary conditions for these models can be found in our

(a) Selected sample VH-IVUS slices.

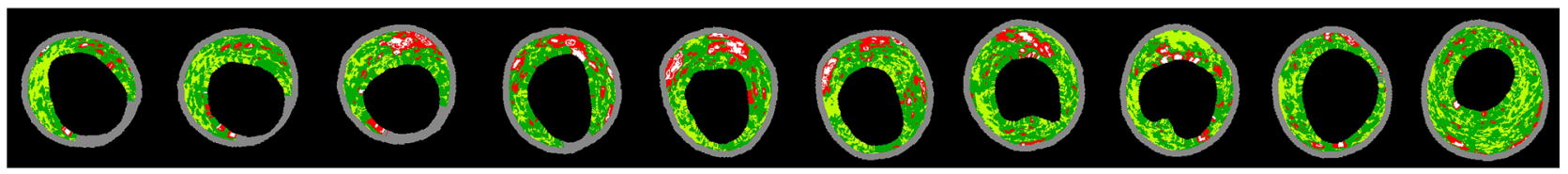

(b) Segmented contours plots.
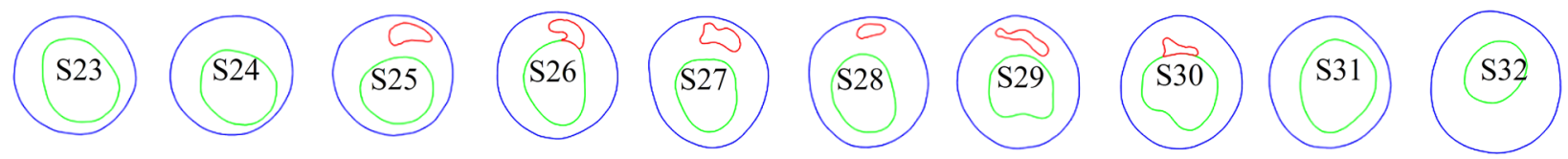

(d) 3D geometry reconstruction of vessel
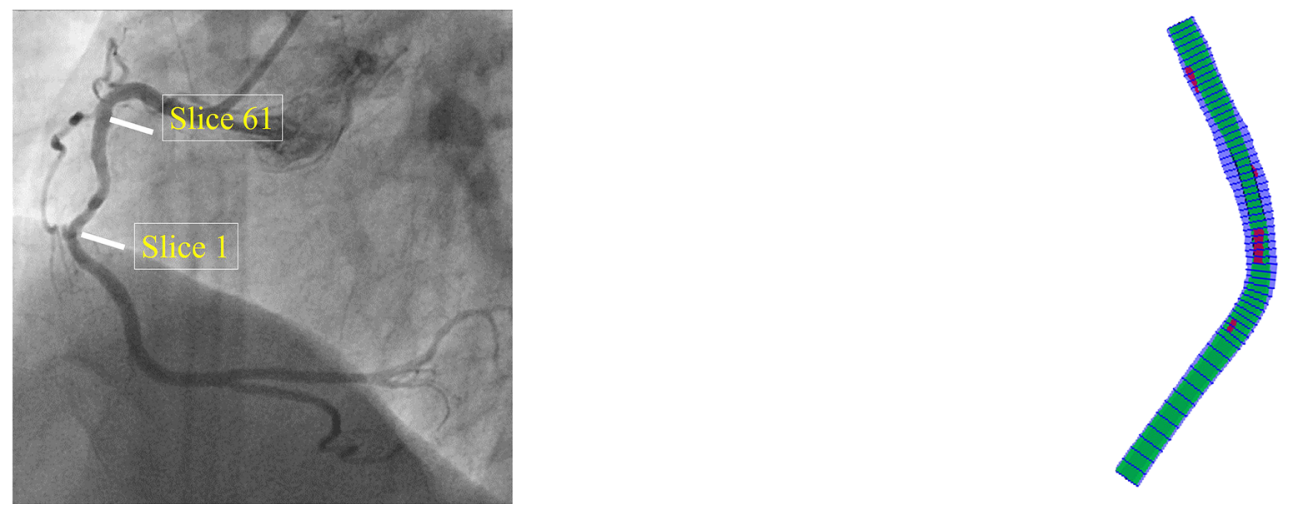

Fig. 1 Selected sample VH-IVUS slices, segmented contour plots, X-ray angiographic image from a patient and the 3D vessel geometry reconstruction. Colors in VH-IVUS images: red, lipid; white, calcification; dark green, fibrous; light green, fibro-fatty

Table 2 List of models used in this paper ("Y": feature included; "N": feature not included)

\begin{tabular}{lllll}
\hline Models & $\begin{array}{l}\text { Circumferential } \\
\text { shrink process }\end{array}$ & Axial shrink & $\begin{array}{l}\text { Cyclic } \\
\text { bending }\end{array}$ & FSI \\
\hline M1: 2D with circumferential shrink & $\mathrm{Y}$ & $\mathrm{N}$ & $\mathrm{N}$ & $\mathrm{N}$ \\
M2: 2D without circumferential shrink & $\mathrm{N}$ & $\mathrm{N}$ & $\mathrm{N}$ & $\mathrm{N}$ \\
M3: 3D FSI model with cyclic bending & $\mathrm{Y}$ & $\mathrm{Y}$ & $\mathrm{Y}$ & $\mathrm{Y}$ \\
M4: 3D FSI model without cyclic bending & $\mathrm{Y}$ & $\mathrm{Y}$ & $\mathrm{N}$ & $\mathrm{Y}$ \\
M5: 3D TL structure-only model & $\mathrm{Y}$ & $\mathrm{Y}$ & $\mathrm{N}$ & $\mathrm{N}$ \\
M6: 3D structure-only vessel model & $\mathrm{Y}$ & $\mathrm{Y}$ & $\mathrm{Y}$ & $\mathrm{N}$ \\
M7: 3D fluid-only vessel model & $\mathrm{N}$ & $\mathrm{N}$ & $\mathrm{N}$ & $\mathrm{N}$ \\
\hline
\end{tabular}


previous publication (Wang $\mathrm{H}$ et al. 2015; Wang Q et al. 2017; Yang et al. 2009). These models were selected in this paper to compare and investigate the impact of circumferential shrink, axial shrink, FSI and FSI with cyclic bending on model solution behaviors (plaque stress/strain and flow shear stress). The FSI model with cyclic bending (M3) is the best approximation to the vessel physical reality among the seven models and was used as the gold standard when applicable. For this model, blood flow was assumed to be laminar, viscous, incompressible and Newtonian. The incompressible Navier-Stokes equations with arbitrary Lagrangian-Eulerian (ALE) formulation were used as the governing equations. Physiological pressure conditions were prescribed at both inlet and outlet. Noslip conditions and natural traction equilibrium conditions were assumed at all interfaces between fluid and vessel and between plaque components. Putting these together, we have (summation convention is used):

$\rho\left(\partial \mathbf{u} / \partial t+\left(\left(\mathbf{u}-\mathbf{u}_{g}\right) \cdot \nabla\right) \mathbf{u}\right)=-\nabla p+\mu \nabla^{2} \mathbf{u}$

$\nabla \cdot \mathbf{u}=0$

$\left.\mathbf{u}\right|_{\Gamma}=\partial \mathbf{x} / \partial t, \partial \mathbf{u} /\left.\partial n\right|_{\text {inlet,outlet }}=0$

$\left.p\right|_{\text {inlet }}=p_{\text {in }}(t),\left.p\right|_{\text {outlet }}=p_{\text {out }}(t),\left.p\right|_{\text {out boundary }}=0$,

$\rho v_{i, t t}=\sigma_{i j, j}, i, j=1,2,3$, sum overj,

$\varepsilon_{i j}=v_{i, j}+v_{j, i}+v_{\alpha, i} v_{\alpha, j} / 2, i, j, \alpha=1,2,3$

$\left.\sigma_{i, j} \bullet n_{j}\right|_{\text {out }_{\text {wall }}}=0$

$\left.\sigma_{i j}^{r} \cdot n_{j}\right|_{\text {interface }}=\left.\sigma_{i j}^{s} \cdot n_{j}\right|_{\text {interface }}$,

$\mathbf{x}_{\text {center }}=\mathbf{x}_{\text {bending }}(t)$,

where $\mathbf{u}$ and $p$ are fluid velocity and pressure, $\mathbf{x}_{\text {center }}$ is the vessel centerline, $\mathbf{x}_{\text {bending }}$ is the imposed cyclic bending condition derived from patient angiography movie, $\mathbf{u}_{\mathrm{g}}$ is the mesh velocity, $\mu$ is the dynamic viscosity, $\rho$ is density, $\Gamma$ stands for vessel inner boundary, $f_{\bullet}, j$ stands for derivative of $f$ with respect to the $j$ th variable, $\sigma$ is the stress tensor (superscripts indicate different materials), $\varepsilon$ is the strain tensor, $\mathbf{v}$ is the solid displacement vector, superscript letters $r$ and $s$ were used to indicate different materials (fluid or different plaque components). For simplicity, all material densities were set to 1 in this paper.

\subsubsection{The anisotropic Mooney-Rivlin model for material properties and parameter values}

Coronary vessel material was assumed to be hyperelastic, anisotropic, nearly-incompressible and homogeneous. The anisotropic Mooney-Rivlin model was used to describe the material properties of the vessel tissue (Bathe 2002). The strain energy density function was:

$$
\begin{aligned}
W= & c_{1}\left(I_{1}-3\right)+c_{2}\left(I_{2}-3\right)+D_{1}\left[\exp \left(D_{2}\left(I_{1}-3\right)\right)-1\right] \\
& +\left(K_{1} / K_{2}\right) \exp \left[K_{2}\left(I_{4}-1\right)^{2}-1\right], \\
\mathrm{I}_{1}= & \sum C_{i i}, \quad \mathrm{I}_{2}=1 / 2\left[I_{1}^{2}-C_{i j} C_{i j}\right],
\end{aligned}
$$

where $I_{1}$ and $I_{2}$ are the first and second invariants of right Cauchy-Green deformation tensor $\mathbf{C}$ defined as $\boldsymbol{C}=\left[C_{i j}\right]=\mathbf{X}^{\mathbf{T}} \mathbf{X}, \mathbf{X}=\left[\mathrm{X}_{\mathrm{ij}}\right]=\left[\partial \mathrm{x}_{\mathrm{i}} / \partial \mathrm{a}_{\mathrm{j}}\right],\left(\mathrm{x}_{\mathrm{i}}\right)$ is current position, $\left(\mathrm{a}_{\mathrm{i}}\right)$ is original position, $\mathrm{I}_{4}=C_{i j}\left(\mathbf{n}_{c}\right)_{i}\left(\mathbf{n}_{c}\right)_{j}, \mathbf{n}_{c}$ is the unit vector in the circumferential direction of the vessel, $c_{1}, c_{2}$, $\mathrm{D}_{1}, \mathrm{D}_{2}, \mathrm{~K}_{1}$ and $\mathrm{K}_{2}$ are material parameters. Plaque components were assumed to be isotropic, and the isotropic Mooney-Rivlin material model was used to describe their material properties:

$W=c_{1}\left(I_{1}-3\right)+c_{2}\left(I_{2}-3\right)+D_{1}\left[\exp \left(D_{2}\left(I_{1}-3\right)\right)-1\right]$,

In this paper, the following parameter values were chosen: vessel tissue/fibrous cap, $c_{1}=-1312.9 \mathrm{kPa}$, $c_{2}=114.7 \mathrm{kPa}, D_{1}=629.7 \mathrm{kPa}, D_{2}=2.0, K_{1}=35.9 \mathrm{kPa}$, $K_{2}=23.5$; Lipid: $c_{1}=0.5 \mathrm{kPa}, c_{2}=0 \mathrm{kPa}, D_{1}=0.5 \mathrm{kPa}$, $D_{2}=1.5$; Calcification: $c_{1}=92.0 \mathrm{kPa}, c_{2}=0 \mathrm{kPa}$, $D_{1}=36.0 \mathrm{kPa}, D_{2}=2.0$ (Tang et al. 2004; Kural et al. 2012). Axial shrinkage was set at $10 \%$ in our models (if applied) because atherosclerotic vessels are stiffer than healthy vessels. Circumferential pre-shrink process was performed to ensure that the vessel would regain its in vivo geometry when pressure conditions were imposed (Guo et al. 2017). It should be noted that circumferential shrinkage rates were determined by material properties using an iterative process and could not be specified arbitrarily.

\subsection{Model simplifications and comparisons}

Starting from the full 3D FSI model given in Sect. 2.1, various simplifications were made to get the six simplified models. 3D TL model was motivated by the need to build plaque models with reduced labor cost for possible practical clinical implementations. 3D TL model uses a 2D slice and adds a $0.5 \mathrm{~mm}$ thickness so that axial shrink-stretch process could be performed. The difference between 3D TL model and 2D model is that 3D TL model includes 
axial shrinkage, while 2D model does not. Clearly 3D TL model does not have the full 3D plaque structure and vessel curvature which 3D FSI model and structure-only model do have. 3D FSI model includes circumferential and axial shrinkage, while 3D fluid-only vessel model does not. Other model differences between 2D, 3D TL, 3D structure-only, 3D fluid-only and 3D FSI models are self-evident. Uniform pressure conditions were specified on lumen for all 2D, 3D TL and structure-only models:

$\left.p\right|_{\text {lumen }}=p_{\text {in }}(t),\left.p\right|_{\text {out boundary }}=0$,

The modeling process with or without circumferential shrink process was used to quantify the influence of zeroload condition on in vivo image-based 2D models (M1 versus M2). 3D FSI models with or without cyclic bending process were used to study the effect of heart motion for coronary artery (M3 versus M4). The differences between 2D model, 3D TL model and 3D FSI model of different patients were compared to determine a better modeling method (cost-efficient and accurate) for possible practical clinical implementations (M1 and M5 versus M3). It has been long argued that the extra labor cost of 3D FSI models may not be necessary if 3D structure-only or fluid-only models could provide reasonable approximations. Differences between 3D structure-only vessel model and 3D FSI model were compared to explore their differences among different patients (M3 versus M6). With the change of blood pressure, the structure of the vessel wall changes. 3D fluid-only vessel model and 3D FSI model in different patients were compared to explore their differences in flow shear stress (FSS) calculations (M3 versus M7).

\subsection{Mesh generation and solution method}

A component-fitting mesh-generation process (Yang et al. 2009) was used to generate mesh for our models. The finite element models were solved by a commercial finite element software ADINA (Adina R \& D, Watertown, MA, USA) following established procedures (Wang $\mathrm{H}$ et al. 2015; Wang Q et al. 2017; Yang et al. 2009). Mesh analysis was performed by refining mesh density by $10 \%$ until solutions became mesh independent, i.e., changes of subsequent solutions became less than $1 \%$. Three cardiac cycles were simulated for all models, and the solution in the third period was taken as the final results for analysis since the solutions for the second and third cycles became almost identical.

\subsection{Data analysis}

Three hundred and eighty-eight (388) slices from seven patients were evaluable for our study. For each slice, flow shear stress (FSS), plaque stress and strain values from 100 evenly-spaced points at the lumen were obtained for analysis. Since stress and strain are tensors, maximum principal stress and strain values at each lumen point were used as the representative scalar values for easy comparison, and denoted as plaque wall stress (PWS) and strain (PWSn) for convenience. The following notations and formulas were used in our calculations:

$\Delta \mathrm{PWS}_{i}=\left|\mathrm{PWS}_{p, i}-\mathrm{PWS}_{q, i}\right|$

Mean $\Delta \mathrm{PWS}=\sum_{i=1}^{n} \Delta \mathrm{PWS}_{i} / n$

$\operatorname{MeanPWS}_{p}=\sum_{i=1}^{n} \mathrm{PWS}_{p, i} / n$

RelativeError $=$ Mean $\Delta$ PWS $/$ MeanPWS $_{p}$

where $i$ is the point index of lumen points and $\mathrm{PWS}_{p, i}$ and $\mathrm{PWS}_{q, i}$ are the PWS values of the $i$ th point in Model $p$ and Model $q$, respectively. Summation was done at slice, patient, and all patient level as needed for our comparison purpose. " $\mathrm{n}$ " is the total points for the summation being performed. PWSn and FSS calculations were done in the same way.

\section{Results}

\subsection{Construction time of 2D and 3D model}

Table 3 summarizes the time cost of the seven simulation modeling strategies. Currently, the time cost of constructing a 2D model or a 3D TL model for a plaque slice is less than $10 \mathrm{~min}$. The time needed to solve a $2 \mathrm{D}$ model or a 3D TL model was less than 2 min. However, it took more than one week for a trained researcher to construct a 3D FSI model. In addition, more than $10 \mathrm{~h}$ was required to solve a full 3D transient FSI model (Dell Workstation, Precision 5810). The time cost of constructing a

Table 3 Time cost of the seven models

\begin{tabular}{lll}
\hline Models & Building time & Computing time \\
\hline M1 & $<10 \mathrm{~min} /$ slice & $<2 \mathrm{~min} / \mathrm{slice}$ \\
M2 & $<10 \mathrm{~min} / \mathrm{slice}$ & $<2 \mathrm{~min} / \mathrm{slice}$ \\
M3 & $>1$ week/vessel & $>10 \mathrm{~h} / \mathrm{vessel}$ \\
M4 & $>1$ week/vessel & $>10 \mathrm{~h} / \mathrm{vessel}$ \\
M5 & $<10 \mathrm{~min} / \mathrm{slice}$ & $<2 \mathrm{~min} / \mathrm{slice}$ \\
M6 & $>3$ days/vessel & $>5 \mathrm{~h} /$ vessel \\
M7 & $>2$ days/vessel & $>10 \mathrm{~h} /$ vessel \\
\hline
\end{tabular}


3D fluid-only vessel model or a 3D structure-only vessel model was more than 2 or 3 days, respectively. Compared with the 3D FSI model, 2D model and 3D TL model can provide timely plaque mechanics analysis for potential clinical application and commercial implementations.

\subsection{D models without circumferential shrink process produced higher PWS and PWSn values}

To demonstrate model differences with and without circumferential shrinkage, Fig. 2 shows PWS and PWSn plots of one stable plaque (S13 from P1) with no lipid-rich necrotic core (lipid for short) and one unstable plaque (S6 from P5) (a) S13: Stable, In Vivo

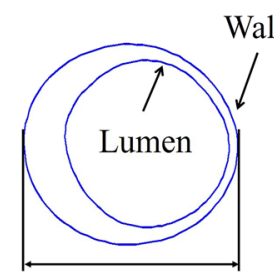

$3.80 \mathrm{~mm}$

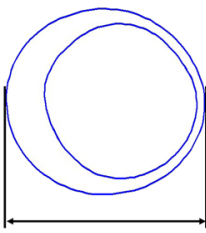

$3.53 \mathrm{~mm}$

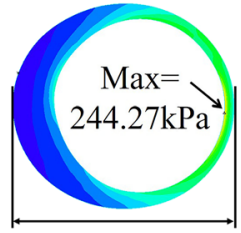

$3.91 \mathrm{~mm}$

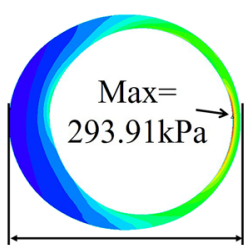

$4.11 \mathrm{~mm}$

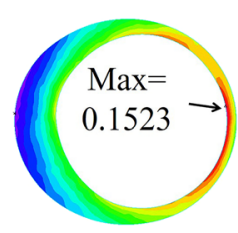

(k) S6-M1: PWSn

(j) S6-M2: PWS No load



$4.292 \mathrm{~mm}$

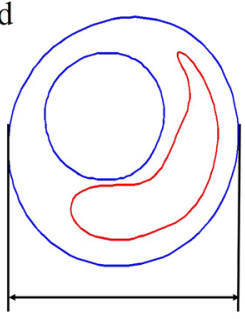

$4.085 \mathrm{~mm}$

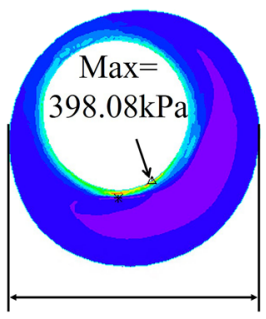

$4.385 \mathrm{~mm}$

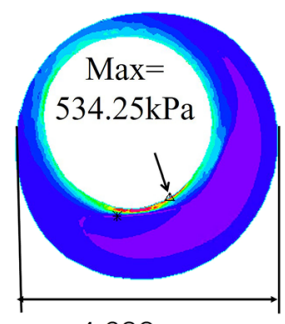

$4.622 \mathrm{~mm}$ (e) S13-M1: PWSn

(f) S13-M2: PWSn
Min
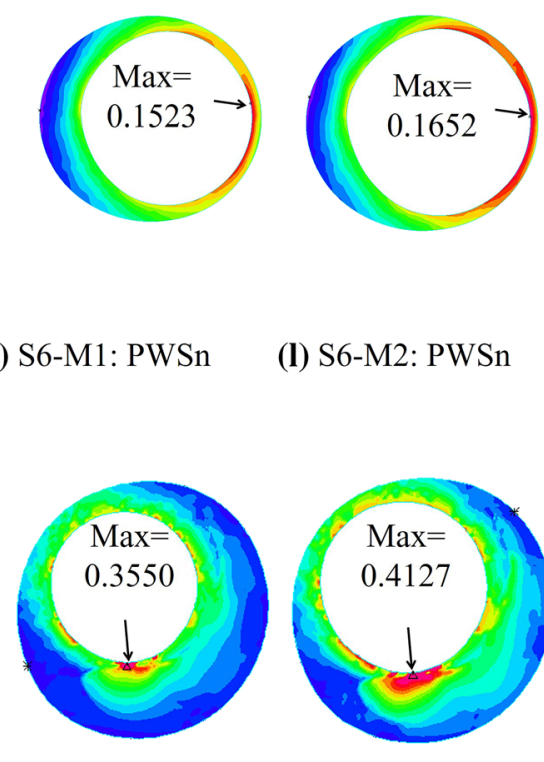

(l) S6-M2: PWSn

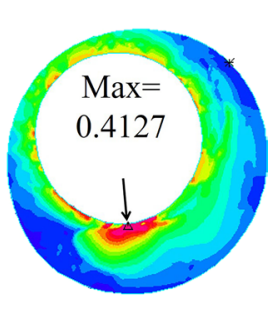

Max

Fig. 2 PWS and PWSn plots of M1 and M2 models showing impact of circumferential shrink process on 2D models. M1: 2D with circumferential shrink; M2: 2D without circumferential shrink

Table 4 PWS, PWSn and errors for seven patients obtained using M1 and M2 models

\begin{tabular}{lcrllllr}
\hline Patients & \multicolumn{2}{l}{ PWS $(\mathrm{kPa})$} & & & \multicolumn{2}{l}{ PWSn } \\
\cline { 2 - 3 } & M1 & M2 & Error (\%) & & M1 & M2 & Error (\%) \\
\hline P1 & 88.6 & 98.5 & 15.40 & & 0.0934 & 0.0969 & 5.85 \\
P2 & 96.0 & 107.9 & 14.31 & & 0.0963 & 0.1001 & 4.93 \\
P3 & 97.0 & 124.0 & 29.35 & & 0.0860 & 0.0945 & 12.46 \\
P4 & 77.4 & 85.5 & 11.13 & & 0.0882 & 0.0918 & 4.64 \\
P5 & 152.5 & 190.5 & $\mathbf{2 9 . 6 5}$ & & 0.1929 & 0.2070 & $\mathbf{1 3 . 4 0}$ \\
P6 & 72.3 & 80.2 & 11.20 & & 0.0846 & 0.0882 & 4.77 \\
P7 & 95.4 & 104.0 & $\mathbf{9 . 7 9}$ & & 0.0987 & 0.1019 & $\mathbf{3 . 8 7}$ \\
Ave & 97.0 & 112.9 & 17.26 & & 0.1057 & 0.1115 & 7.13 \\
\hline
\end{tabular}

M1: 2D with circumferential shrink; M2: 2D without circumferential shrink. Errors were calculated using M1 as the base

The maximum and minimum values of errors from the seven patients were indicated in bold 
with a large lipid-rich necrotic core and a thin fibrous cap from two models (M1 and M2). To observe PWS and PWSn patient variations, Table 4 lists PWS and PWSn mean values from M1 and M2 for each patient and the relative errors of M2 using M1 values as the baseline. Mean PWS and PWSn relative errors were $17.26 \%$ and $7.13 \%$ for the seven patients combined, respectively. PWS relative error patient variations ranged from $9.79 \%$ to $29.65 \%$, while PWSn relative error variation ranged from $3.87-13.40 \%$.

\subsection{Influence of cyclic bending process on coronary artery modeling}

Considering the effect of cardiac motion on coronary arteries, 3D FSI model with cyclic bending (M3) and 3D FSI model without cyclic bending (M4) were constructed. Figure 3 shows the PWS, PWSn and FSS plots from M3 and M4 for P1. Table 5 summarizes PWS and PWSn mean values and maximum FSS (Max-FSS) values from M3 and M4 for each patient. The relative errors of M4 using M3 values as the baseline were given. The average relative error value of (a) P1-M3: $\kappa_{T P}=1.0 \mathrm{~cm}^{-1}$

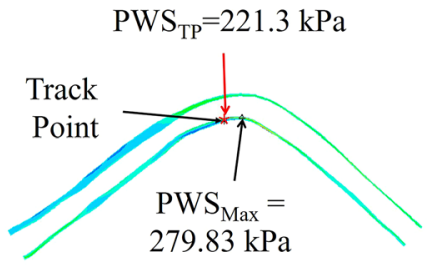

(d) P1-M4: $\kappa_{T P}=0.6 \mathrm{~cm}^{-1}$

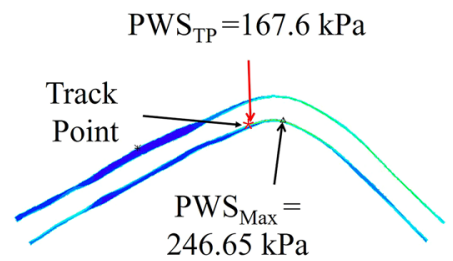

(b) P1-M3: $\kappa_{T P}=1.0 \mathrm{~cm}^{-1}$

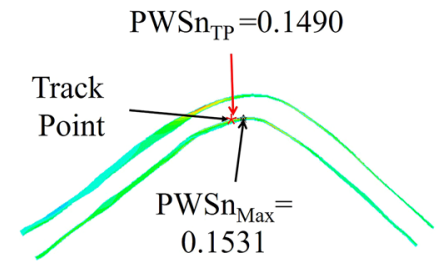

(e) P1-M4: $\kappa_{T P}=0.6 \mathrm{~cm}^{-1}$

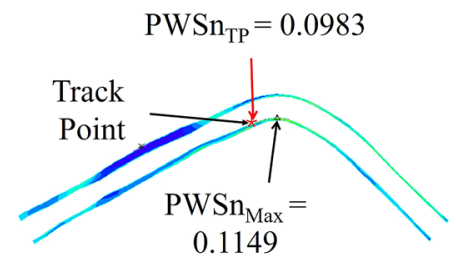

(c) P1-M3: $\kappa_{T P}=1.0 \mathrm{~cm}^{-1}$

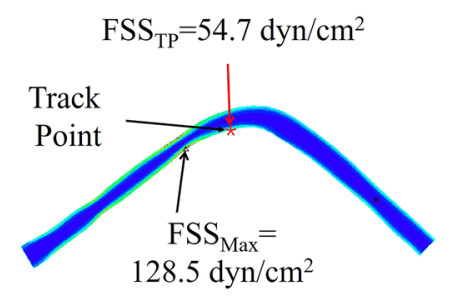

(f) P1-M4: $\kappa_{T P}=0.6 \mathrm{~cm}^{-1}$

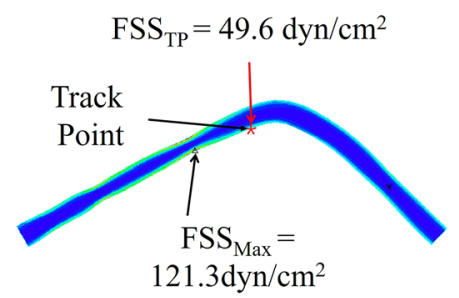

Min $\operatorname{Max}$

Fig. 3 The influence of curvature change of coronary artery with cardiac motion (M3 vs M4) on PWS, PWSn and FSS calculations. M3: 3D FSI model with cyclic bending; M4: 3D FSI model without cyclic bending. TP: tracking point with the greatest curvature change. $\kappa_{T P}$ : curvature at tracking point
Table 5 PWS, PWSn, FSS values from M3 and M4 models for seven patients under maximum pressure condition

\begin{tabular}{|c|c|c|c|c|c|c|c|c|c|}
\hline \multirow[t]{2}{*}{ Patient } & \multicolumn{3}{|c|}{ PWS (kPa) } & \multicolumn{3}{|l|}{ PWSn } & \multicolumn{3}{|c|}{$\operatorname{Max}-\mathrm{FSS}\left(\mathrm{dyn} / \mathrm{cm}^{2}\right)$} \\
\hline & M3 & M4 & Error $(\%)$ & M3 & M4 & Error (\%) & M3 & M4 & Error $(\%)$ \\
\hline P1 & 79.2 & 75.6 & 12.08 & 0.0713 & 0.0628 & 15.61 & 136.9 & 132.7 & 3.04 \\
\hline $\mathrm{P} 2$ & 82.8 & 81.0 & 7.16 & 0.0682 & 0.0634 & 10.29 & 167.4 & 171.0 & 2.13 \\
\hline P3 & 86.4 & 86.5 & 12.26 & 0.0696 & 0.0708 & 13.71 & 36.8 & 37.1 & 0.90 \\
\hline P4 & 71.6 & 66.0 & 13.64 & 0.0721 & 0.0598 & 19.24 & 179.4 & 178.5 & 0.46 \\
\hline P5 & 113.2 & 113.4 & 8.08 & 0.1535 & 0.1438 & 9.48 & 66.3 & 62.1 & 6.29 \\
\hline P6 & 57.6 & 54.5 & 14.88 & 0.0648 & 0.0599 & 15.29 & 223.5 & 228.3 & 2.15 \\
\hline P7 & 84.4 & 78.7 & 12.39 & 0.0776 & 0.0647 & 18.21 & 276.3 & 267.6 & 3.16 \\
\hline Ave & 82.2 & 79.4 & 11.50 & 0.0824 & 0.0750 & 14.55 & 135.1 & 153.6 & 2.59 \\
\hline
\end{tabular}

M3: 3D FSI model with cyclic bending; M4: 3D FSI model without cyclic bending. Errors were calculated using M3 as the base

The maximum and minimum values of errors from the seven patients were indicated in bold 
PWS in M3 and M4 models of seven patients was $11.50 \%$, and the variation range was $7.16 \%-14.88 \%$. The average relative error value of PWSn calculated by M3 and M4 models in seven patients was $14.55 \%$ (ranged from $9.48 \%$ to $19.24 \%$ ). The average relative error value of Max-FSS in M3 and M4 models of seven patients was $2.59 \%$, and the variation range was $0.46 \%-6.29 \%$.

The curvature of the coronary arteries at different locations varies with the cyclic bending process. To observe the influence of curvature change of coronary artery on PWS, PWSn and FSS calculation, curvature changes were tracked at all nodal points of the interface of lumen and the inner surface of the vessel wall. Figure 3 shows the tracking point (TP) with greatest curvature change and provided PWS, PWSn and FSS values at that location. Table 6 lists the PWS, PWSn and FSS values from M3 and M4 models for seven patients at the locations with the greatest curvature change and the relative errors of M4 using M3 values as the baseline. Mean PWS, PWSn and FSS relative errors were $30.13 \%, 23.25 \%$ and $6.75 \%$ for the seven patients combined, respectively. PWS relative error patient variations ranged between $15.07 \%$ and $49.52 \%$, while the PWSn relative error patient variation range was $7.48 \%-34.05 \%$. FSS relative error patient variation range was more moderate at $1.52 \%$ $-12.95 \%$.

\subsection{Comparison of 2D model, 3D TL model and 3D FSI model}

To demonstrate model differences between 2D, 3D TL and 3D FSI models, Fig. 4 provides PWS and PWSn plots of one stable plaque (S13) and one unstable plaque (S6) from three models (M1, M3 and M5). Table 7 lists PWS and PWSn mean values from M1 and M3 for each patient and the relative errors of M1 using M3 values as the baseline. Mean PWS and PWSn relative errors were $33.49 \%$ and $34.18 \%$ for the seven patients combined, respectively. PWS relative error variation range was $18.72-59.54 \%$, while PWSn relative error variation range was $24.04 \%-46.07 \%$. Table 8 lists PWS and PWSn mean values from M5 and M3 for each patient and the relative errors of M5 using M3 values as the baseline. Mean PWS and PWSn relative errors were 22.40\% and $23.08 \%$ for the seven patients combined, respectively. PWS relative error variation range was $14.12-42.24 \%$, while PWSn relative error variation range was $11.80-41.90 \%$. Taking the M3 model as the standard, mean PWS and PWSn relative errors of M5 model of seven patients were 11.09\% and $11.1 \%$ lower than that of M1 model.

\subsection{Comparison of 3D structure-only vessel model and 3D FSI model}

Figure 5 shows the PWS and PWSn plots of P1 from M3 and M6 models to demonstrate model differences between 3D FSI and 3D structure-only models. Table 9 summarizes PWS and PWSn mean values from M3 and M6 for each patient and the relative errors of M6 using M3 values as the baseline. Mean PWS and PWSn relative errors were $4.38 \%$ and $1.78 \%$ for the seven patients combined, respectively. PWS relative error variation range was $0.29-8.27 \%$, while PWSn relative error variation range was $0.22-3.17 \%$.

\subsection{Comparison of 3D fluid-only vessel model and 3D FSI model}

Figure 6 shows the FSS plots under maximum and minimum pressure conditions from M3 and M7 show computational differences between M3 and M7 models of P5. Table 10 summarizes the maximum FSS (Max-FSS), minimum FSS (Min-FSS) and average FSS (Ave-FSS) from M3 and M7 for each patient under maximum and minimum pressure conditions $\left(\mathrm{P}_{\max }\right.$ and $\left.\mathrm{P}_{\min }\right)$ and the averages over time. The relative errors of M7 using M3 values as the baseline were given. Mean Max-FSS, Min-FSS and AveFSS relative errors in $\mathrm{M} 3$ and $\mathrm{M} 7$ models under $\mathrm{P}_{\max }$ were $4.02 \%, 11.29 \%$ and $5.45 \%$ for the 7 patients combined, respectively. Max-FSS relative error patient variations
Table 6 PWS, PWSn and FSS values from M3 and M4 models for seven patients at the locations with the greatest curvature change and the relative errors of M4 using M3 values as the baseline

\begin{tabular}{|c|c|c|c|c|c|c|c|c|c|}
\hline \multirow[t]{2}{*}{ Patient } & \multicolumn{3}{|c|}{ PWS (kPa) } & \multicolumn{3}{|l|}{ PWSn } & \multicolumn{3}{|c|}{$\mathrm{FSS}\left(\mathrm{dyn} / \mathrm{cm}^{2}\right)$} \\
\hline & M3 & M4 & Error (\%) & M3 & M4 & Error (\%) & M3 & M4 & Error $(\%)$ \\
\hline $\mathrm{P} 1$ & 221.3 & 167.6 & 24.27 & 0.1490 & 0.0983 & 34.05 & 54.7 & 49.6 & 9.36 \\
\hline $\mathrm{P} 2$ & 290.5 & 163.2 & 43.82 & 0.1240 & 0.0947 & 23.68 & 41.8 & 36.4 & 12.95 \\
\hline P3 & 129.5 & 103.4 & 20.13 & 0.1174 & 0.1332 & 13.49 & 27.6 & 28.9 & 5.02 \\
\hline P4 & 108.1 & 54.6 & 49.52 & 0.0770 & 0.0570 & 26.00 & 139.1 & 126.1 & 9.38 \\
\hline P5 & 169.9 & 195.6 & 15.07 & 0.1734 & 0.1864 & 7.48 & 47.5 & 46.8 & 1.52 \\
\hline P6 & 112.4 & 92.6 & 17.54 & 0.1026 & 0.0734 & 28.49 & 59.3 & 57.0 & 3.82 \\
\hline P7 & 262.5 & 156 & 40.57 & 0.1399 & 0.0986 & 29.53 & 123.0 & 116.6 & 5.19 \\
\hline Ave & 184.9 & 133.3 & 30.13 & 0.1262 & 0.1059 & 23.25 & 70.4 & 65.9 & 6.75 \\
\hline
\end{tabular}

The maximum and minimum values of errors from the seven patients were indicated in bold 
(a) S13-M1: PWS

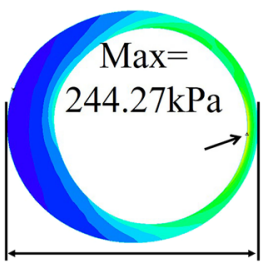

$3.91 \mathrm{~mm}$

(e) S13-M5: PWS

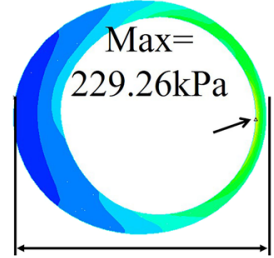

$3.947 \mathrm{~mm}$

(i) S13-M3: PWS

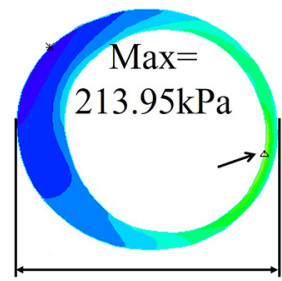

$4.072 \mathrm{~mm}$ (b) S13-M1: PWSn

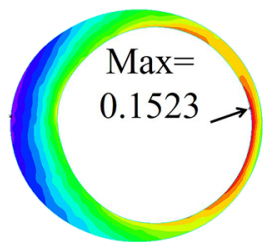

(f) S13-M5: PWSn

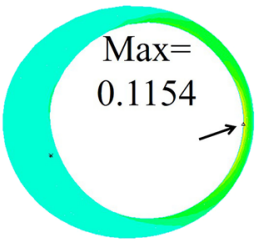

(j) S13- M3: PWSn

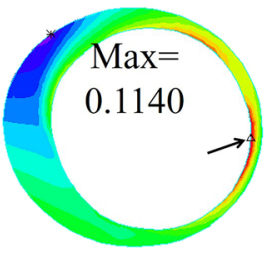

(c) S6-M1: PWS

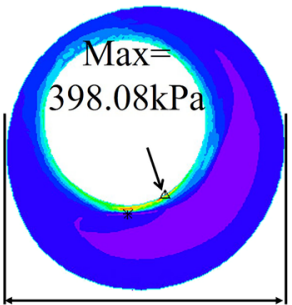

$4.385 \mathrm{~mm}$

(g) S6-M5: PWS

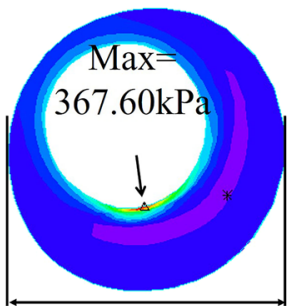

$4.348 \mathrm{~mm}$

(k) S6- M3: PWS



$4.495 \mathrm{~mm}$ (d) S6-M1: PWSn

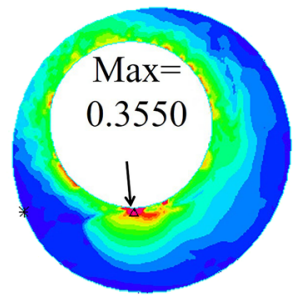

(h) S6-M5: PWSn

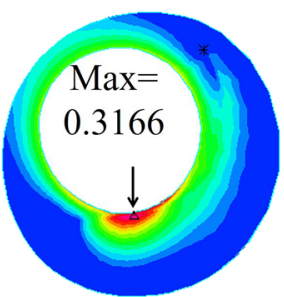

(I) S6-M3: PWSn

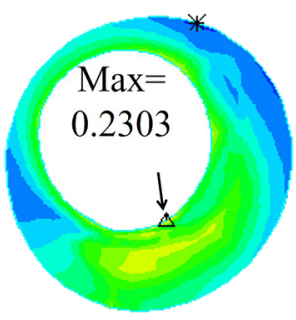

Min

$\operatorname{Max}$

Fig. 4 PWS and PWSn plots from M1, M3 and M5 showing PWS and PWSn differences between 2D, 3D TL and 3D FSI models. M1: 2D with circumferential shrink; M3: 3D FSI model with cyclic bending; M5: 3D TL structure-only model

Table 7 PWS, PWSn from M1 and M3 for seven patients and errors of M1 were calculated using M3 values as the baseline

\begin{tabular}{|c|c|c|c|c|c|c|}
\hline \multirow[t]{2}{*}{ Patient } & \multicolumn{3}{|c|}{ PWS (kPa) } & \multicolumn{3}{|l|}{ PWSn } \\
\hline & M1 & M3 & Error (\%) & M1 & M3 & Error $(\%)$ \\
\hline P1 & 88.61 & 79.24 & 21.33 & 0.0934 & 0.0713 & 32.91 \\
\hline $\mathrm{P} 2$ & 96.00 & 82.79 & 20.30 & 0.0963 & 0.0682 & 41.17 \\
\hline P3 & 97.02 & 86.35 & 59.54 & 0.0860 & 0.0696 & 46.07 \\
\hline P4 & 77.41 & 71.55 & 18.72 & 0.0882 & 0.0721 & 24.04 \\
\hline P5 & 152.45 & 113.22 & 48.74 & 0.1929 & 0.1535 & 27.94 \\
\hline P6 & 72.33 & 57.56 & 43.81 & 0.0846 & 0.0648 & 38.50 \\
\hline P7 & 95.42 & 84.40 & 21.99 & 0.0987 & 0.0776 & 28.61 \\
\hline Ave & 97.03 & 82.16 & 33.49 & 0.1057 & 0.0824 & 34.18 \\
\hline
\end{tabular}

M1: 2D with circumferential shrink; M3: 3D FSI model with cyclic bending

The maximum and minimum values of errors from the seven patients were indicated in bold 
Table 8 PWS, PWSn values from M3 and M5 for seven patients

\begin{tabular}{llllllll}
\hline Patient & \multicolumn{1}{l}{ PWS $(\mathrm{kPa})$} & \multicolumn{2}{l}{ PWSn } \\
\cline { 2 - 3 } & M5 & M3 & Error $(\%)$ & & M5 & M3 & Error (\%) \\
\hline P1 & 82.40 & 79.24 & $\mathbf{1 4 . 1 2}$ & & 0.0640 & 0.0713 & 14.58 \\
P2 & 90.89 & 82.79 & 15.03 & & 0.0674 & 0.0682 & $\mathbf{1 1 . 0 8}$ \\
P3 & 82.93 & 86.35 & $\mathbf{4 2 . 2 4}$ & & 0.0693 & 0.0696 & 35.78 \\
P4 & 72.75 & 71.55 & 16.50 & & 0.0592 & 0.0721 & 19.72 \\
P5 & 96.94 & 113.22 & 22.48 & & 0.1756 & 0.1535 & 19.17 \\
P6 & 68.79 & 57.56 & 29.86 & & 0.0777 & 0.0648 & $\mathbf{4 1 . 9 0}$ \\
P7 & 82.27 & 84.40 & 16.54 & & 0.0636 & 0.0776 & 19.30 \\
Ave & 82.42 & 82.16 & 22.40 & & 0.0824 & 0.0824 & 23.08 \\
\hline
\end{tabular}

M3: 3D FSI model with cyclic bending; M5: 3D TL structure-only model. Errors of M5 were calculated using M3 values as the baseline

The maximum and minimum values of errors from the seven patients were indicated in bold ranged between $0.56 \%$ and $9.52 \%$, while Min-FSS relative error patient variation range was 3.59\%-36.63\%. Ave-FSS relative error patient variation range was more moderate at 4.87-6.25\%. Mean Max-FSS, Min-FSS and Ave-FSS relative errors in $\mathrm{M} 3$ and $\mathrm{M} 7$ models under $\mathrm{P}_{\min }$ were $3.09 \%, 10.17 \%$ and $1.85 \%$ for the seven patients combined, respectively. Max-FSS relative error patient variations ranged between $0.19 \%$ and $7.54 \%$, while Min-FSS relative error patient variation range was $2.83-23.80 \%$. Ave-FSS (a) P1-M3: PWS, $\kappa=1.38 \mathrm{~cm}^{-1}$

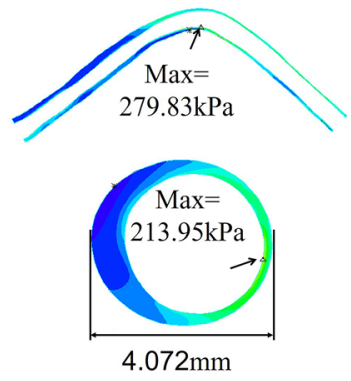

(c) P1- M6: PWS, $\kappa=1.38 \mathrm{~cm}^{-1}$

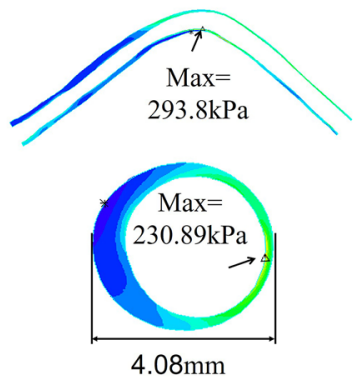

Min (b) P1- M3: PWSn, $\kappa=1.38 \mathrm{~cm}^{-1}$

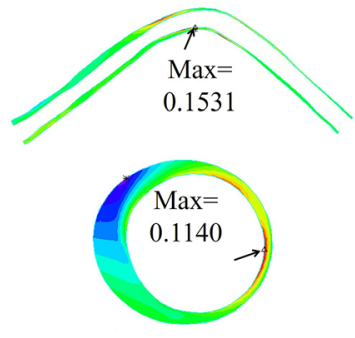

(d) P1- M6: PWSn, $\kappa=1.38 \mathrm{~cm}^{-1}$

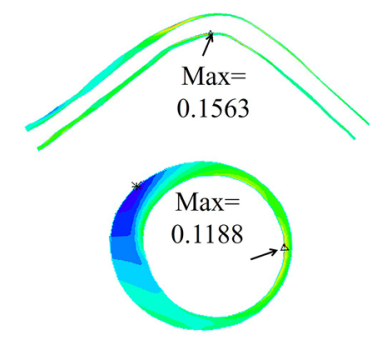

Fig. 5 PWS and PWSn plots from M3 and M6 showing computational differences between 3D structure-only model and 3D FSI models. M3: 3D FSI model with cyclic bending; M6: 3D structure-only vessel model relative error patient variation range was more moderate at $0.38-2.85 \%$. Mean Max-FSS, Min-FSS and Ave-FSS relative errors in M3 and M7 models under the averages over time were $3.44 \%, 9.61 \%$ and $2.88 \%$ for the seven patients combined, respectively. Max-FSS relative error patient variations ranged between 0.62\%-7.94\%, while Min-FSS relative error patient variation range was $1.13-30.15 \%$. Ave-FSS relative error patient variation range was more moderate at $2.33-3.29 \%$.

\section{Discussion}

\subsection{The importance of studying multiple patients}

It is well accepted that different models may provide different computational results. It is also well known that patientspecific modeling and mechanical information are important for diagnosis and precision medicine where medication and treatment strategies would be decided on a patient-by-patient basis. Previous model comparison studies often use singlepatient data or idealized geometries and their results normally report "Model A over-estimates PWS by $30 \%$ compared with Model B (the gold standard)." Holzapfel et al. (2002) introduced a layer-specific 3D anisotropic model (baseline model, or the gold standard) based on in vitro magnetic resonance imaging of a human stenotic postmortem artery. Model differences between the baseline model and other three simplified models (model without axial prestretch, model with plane strain and isotropic model) went as high as 600\%. Yang et al. (2009) compared maximum of Stress- $\mathrm{P}_{1}$ (maximum principal stress) on a cut surface of from five different models using one patient data. Compared to the isotropic model (Model 1, no bending, no axial stretch), maximum Stress- $\mathrm{P}_{1}$ values on the cut surface with maximum bending (where applicable) from Model 2 (anisotropic, no bending, no stretch), Model 3 (anisotropic, with 
Table 9 PWS, PWSn values from M3 and M6 for seven patients
Fig. 6 FSS plots under maximum and minimum pressure conditions changed from M3 and M7 show computational differences between 3D FSI model and 3D fluid-only models. M3: 3D FSI model with cyclic bending; M7: 3D fluid-only vessel model. TP: tracking point with the greatest curvature change. $\kappa_{T P}$ : curvature at tracking point

\begin{tabular}{llllllll}
\hline Patient & \multicolumn{1}{l}{ PWS $(\mathrm{kPa})$} & & & \multicolumn{2}{l}{ PWSn } \\
\cline { 2 - 3 } & M3 & M6 & Error (\%) & & M3 & M6 & Error (\%) \\
\hline P1 & 79.24 & 83.24 & 5.04 & & 0.0713 & 0.0728 & 2.09 \\
P2 & 82.79 & 87.00 & 5.09 & & 0.0682 & 0.0697 & 2.15 \\
P3 & 86.35 & 86.61 & $\mathbf{0 . 2 9}$ & & 0.0696 & 0.0698 & $\mathbf{0 . 2 2}$ \\
P4 & 71.55 & 74.34 & 3.90 & & 0.0721 & 0.0732 & 1.54 \\
P5 & 113.22 & 115.01 & 1.61 & & 0.1535 & 0.1542 & 0.60 \\
P6 & 57.56 & 62.32 & $\mathbf{8 . 2 7}$ & & 0.0648 & 0.0665 & 2.70 \\
P7 & 84.40 & 89.49 & 6.46 & & 0.0776 & 0.0797 & $\mathbf{3 . 1 7}$ \\
Ave & 82.16 & 85.43 & 4.38 & & 0.0824 & 0.0837 & 1.78 \\
\hline
\end{tabular}

M3: 3D FSI model with cyclic bending; M6: 3D structure-only vessel model. Errors of M6 were calculated using M3 values as the baseline

The maximum and minimum values of errors from the seven patients were indicated in bold

(a) P5-M3: Time $=0.4 \mathrm{~s}, \kappa_{T P}=0.3 \mathrm{~cm}^{-1}$

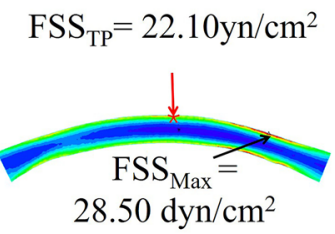

(c) P5-M3: Time $=0.68 \mathrm{~s}, \kappa_{T P}=0.82 \mathrm{~cm}^{-1}$ $\mathrm{FSS}_{\mathrm{TP}}=47.53 \mathrm{yn} / \mathrm{cm}^{2}$

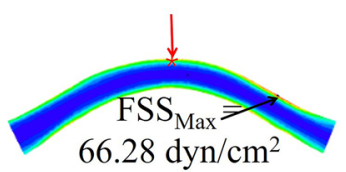

(b) P5-M7: Time $=0.4 \mathrm{~s}, \boldsymbol{\kappa}_{T P}=0.3 \mathrm{~cm}^{-1}$

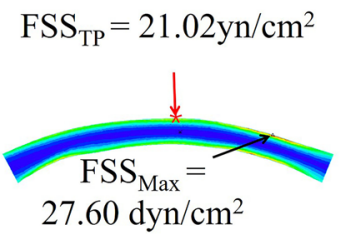

(d) P5-M7: Time $=0.68 \mathrm{~s}, \kappa_{T P}=0.3 \mathrm{~cm}^{-1}$

$\mathrm{FSS}_{\mathrm{TP}}=44.79 \mathrm{yn} / \mathrm{cm}^{2}$

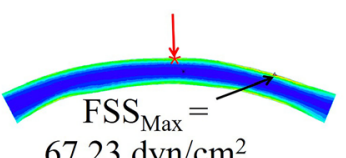

$67.23 \mathrm{dyn} / \mathrm{cm}^{2}$

Min

Max

bending, no stretch) and Model 4 (anisotropic with bending and stretch) were 63\%, 126\% and 345\% higher than that from Model 1, respectively. Guo et al. used in vivo vessel material properties in their coronary plaque models and they reported that average cap strain values using in vivo material models were 150\%-180\% higher than those from the ex vivo material models. The corresponding percentages for average cap stress values were 50-75\% (Guo et al. 2017). Wang L. et al. used a near-idealized plaque geometry to study the impact of residual stress (opening angle), axial shrink-stretch and circumferential pre-shrink on plaque stress/strain calculations. They reported that the model with axial stretch, circumferential shrink, but omitting opening angle overestimated lumen and cap stress by $182 \%$ and $448 \%$, respectively (Wang L. et al. 2017). While the large percentage values demonstrated the importance of using the "right" models for plaque mechanical analysis, it is natural to move on to multi-patient studies to investigate patient variations in model differences. Table 11 lists model comparison difference ranges reported in this paper. For seven patients, PWS difference from 2D models with/without circumferential shrink process varied from 9.79 to $29.65 \%$. PWS change at location with greatest curvature change from 3D FSI models with/without cyclic bending varied from 15.07 to $49.52 \%$. As for hydrodynamic calculation difference analysis, the Min-FSS difference between 3D fluid-only vessel models and 3D FSI models varied from 3.59 to $36.63 \%$ for seven patients. Patient variations for other model comparisons gave similar results. Caution should be taken when interpreting computational results from different models, with patient variations kept in mind. 
Table 10 FSS values from M3 and $\mathrm{M} 7$ for seven patients

\begin{tabular}{|c|c|c|c|c|c|c|c|c|c|}
\hline \multirow[t]{2}{*}{ Patient } & \multicolumn{3}{|c|}{ Max-FSS (dyn/cm²) } & \multicolumn{3}{|c|}{ Min-FSS $\left(\mathrm{dyn} / \mathrm{cm}^{2}\right)$} & \multicolumn{3}{|c|}{ Ave-FSS $\left(\mathrm{dyn} / \mathrm{cm}^{2}\right)$} \\
\hline & M3 & M7 & Error $(\%)$ & M3 & M7 & Error $(\%)$ & M3 & M7 & Error $(\%)$ \\
\hline \multicolumn{10}{|c|}{ Under maximum pressure condition } \\
\hline P1 & 136.9 & 141.0 & 3.03 & 14.9 & 15.8 & 6.13 & 54.4 & 57.3 & 5.27 \\
\hline $\mathrm{P} 2$ & 167.4 & 172.2 & 2.83 & 12.6 & 14.3 & 13.93 & 51.6 & 53.3 & 4.87 \\
\hline P3 & 36.8 & 39.8 & 8.41 & 6.4 & 6.0 & 5.66 & 19.1 & 19.7 & 5.38 \\
\hline P4 & 179.4 & 183.5 & 2.33 & 21.1 & 22.6 & 7.03 & 66.9 & 70.9 & 6.06 \\
\hline P5 & 66.3 & 67.23 & 1.43 & 12.2 & 16.7 & 36.63 & 35.0 & 36.8 & 5.03 \\
\hline P6 & 223.5 & 244.8 & 9.52 & 18.4 & 19.0 & 3.59 & 77.5 & 79.6 & 5.29 \\
\hline P7 & 276.3 & 277.9 & 0.56 & 15.2 & 16.1 & 6.05 & 94.4 & 97.8 & 6.25 \\
\hline Ave & 135.1 & 160.9 & 4.02 & 14.4 & 15.8 & 11.29 & 57.0 & 59.3 & 5.45 \\
\hline \multicolumn{10}{|c|}{ Under minimum pressure condition } \\
\hline $\mathrm{P} 1$ & 43.7 & 44.7 & 2.21 & 6.1 & 6.4 & 5.45 & 18.8 & 19.0 & 1.11 \\
\hline $\mathrm{P} 2$ & 55.0 & 55.1 & 0.19 & 5.2 & 5.8 & 11.93 & 17.4 & 17.8 & 2.26 \\
\hline P3 & 19.4 & 20.8 & 7.54 & 4.2 & 3.8 & 9.75 & 10.7 & 10.9 & 2.04 \\
\hline P4 & 35.9 & 36.6 & 1.96 & 5.8 & 6.2 & 6.70 & 13.8 & 14.1 & 2.31 \\
\hline P5 & 28.5 & 27.6 & 3.20 & 7.0 & 8.7 & 23.80 & 17.2 & 17.2 & 0.38 \\
\hline P6 & 47.8 & 49.2 & 2.86 & 5.5 & 5.4 & 2.83 & 16.1 & 16.5 & 1.97 \\
\hline P7 & 95.9 & 99.4 & 3.68 & 8.9 & 7.9 & 10.74 & 35.5 & 36.5 & 2.85 \\
\hline Ave & 46.6 & 47.6 & 3.09 & 6.1 & 6.3 & 10.17 & 18.5 & 18.9 & 1.85 \\
\hline \multicolumn{10}{|c|}{ Average over cardiac cycle } \\
\hline P1 & 108.5 & 111.5 & 2.76 & 12.9 & 13.6 & 5.73 & 44.2 & 45.3 & 2.51 \\
\hline $\mathrm{P} 2$ & 134.9 & 136.9 & 1.48 & 10.8 & 12.0 & 11.11 & 41.6 & 42.8 & 3.06 \\
\hline P3 & 28.4 & 30.6 & 7.94 & 5.4 & 5.0 & 7.41 & 15.1 & 15.5 & 2.33 \\
\hline P4 & 120.8 & 123.3 & 2.10 & 15.4 & 16.4 & 6.78 & 45.3 & 46.7 & 3.15 \\
\hline P5 & 45.0 & 45.3 & 0.62 & 10.1 & 13.1 & 30.15 & 26.8 & 27.7 & 3.29 \\
\hline P6 & 175.0 & 188.2 & 7.58 & 15.3 & 16.0 & 4.96 & 59.8 & 61.3 & 2.57 \\
\hline P7 & 231.5 & 235.2 & 1.61 & 13.9 & 13.8 & 1.13 & 80.6 & 83.2 & 3.24 \\
\hline Ave & 120.6 & 124.4 & 3.44 & 12.0 & 12.8 & 9.61 & 44.8 & 46.1 & 2.88 \\
\hline
\end{tabular}

M3: 3D FSI model with cyclic bending; M7: 3D fluid-only vessel model. Errors of M7 were calculated using M3 values as the baseline

The maximum and minimum values of errors from the seven patients were indicated in bold

Table 11 Summary of model comparison patient variation ranges

\begin{tabular}{|c|c|c|c|c|c|}
\hline Models compared & PWS (\%) & PWSn (\%) & Max-FSS (\%) & Min-FSS (\%) & Ave-FSS (\%) \\
\hline 2D models with/without circumferential shrink (M1 vs. M2) & $9.79-29.65$ & $3.87-13.40$ & N/A & N/A & N/A \\
\hline 3D FSI models with/without cyclic bending (M3 vs. M4) & $7.16-14.88$ & $9.48-19.24$ & $0.46-6.29$ & N/A & N/A \\
\hline $\begin{array}{l}\text { 3D FSI models with/without cyclic bending at locations with max } \\
\text { curvature change (M3 vs. M4) }\end{array}$ & $15.07-49.52$ & $7.48-34.05$ & $1.52-12.95$ & N/A & N/A \\
\hline 2D model vs. 3D TL model (M1 vs. M3) & $18.72-59.54$ & $24.04-46.07$ & N/A & N/A & N/A \\
\hline 3D TL model vs. 3D FSI model (M3 vs. M5) & $14.12-42.24$ & $11.08-41.90$ & N/A & N/A & N/A \\
\hline 3D structure-only model versus 3D FSI model (M3 vs. M6) & $0.29-8.27$ & $0.22-3.17$ & N/A & N/A & N/A \\
\hline $\begin{array}{l}\text { 3D fluid-only model versus 3D FSI model (M3 vs. M7), maximum } \\
\text { pressure }\end{array}$ & N/A & N/A & $0.56-9.52$ & $3.59-36.63$ & $4.87-6.25$ \\
\hline $\begin{array}{l}\text { 3D fluid-only model versus 3D FSI model (M3 vs. M7), minimum } \\
\text { pressure condition }\end{array}$ & N/A & N/A & $0.19-7.54$ & $2.83-23.80$ & $0.38-2.85$ \\
\hline $\begin{array}{l}\text { 3D fluid-only model versus 3D FSI model (M3 vs. M7), Average over } \\
\text { a cardiac cycle }\end{array}$ & N/A & N/A & $0.62-7.94$ & $1.13-30.15$ & $2.33-3.29$ \\
\hline
\end{tabular}

Numbers given below are percentages of model solution differences unless otherwise indicated. N/A: not applicable 


\subsection{The importance of pre-shrink process for image-based models}

As shown in Table 4, PWS and PWSn values of the plaques would be overestimated under the models without shrink process. The influence of 2D model without shrink process on PWS calculation is greater than on PWSn calculations. The errors of PWS and PWSn were $17.26 \%$ and $7.13 \%$, respectively. Patient variations of PWS errors for the seven patients ranged from 9.79 to $29.65 \%$. This demonstrates that pre-shrinking process and correct initial zero-load geometry are important for models based on in vivo images to obtain accurate computational stress/strain results.

\subsection{The influence of cyclic bending on the results of coronary artery simulation modeling}

The cyclic bending process has great influence on 3D FSI model calculations, especially at locations with large curvature changes. PWS and PWSn values from 3D FSI models with and without cyclic bending for seven patients showed significant differences at the locations with the greatest curvature change (mean PWS and PWSn relative errors were $30.13 \%$ and $23.25 \%$, respectively). There were large patient variations in those errors (PWS: 15.07\%-49.52\%; PWSn: $7.48-34.05 \%)$. Clearly, the effect of heart movement on the coronary plaque model needs to be considered. Cyclic bending is only one step in fully coupling heart motion to plaque models. Fully coupled ventricle-vessel FSI models should be considered in the future.

\subsection{D TL model was superior to the $2 \mathrm{D}$ model}

A major limitation of 3D FSI models is the model construction time cost. Compared with 3D FSI models, 2D and 3D TL models have much lower time cost (less than $10 \mathrm{~min}$ for each slice). Considering their simplicity and low time consumption, 2D and 3D TL models are more suitable for clinical simulation modeling than 3D FSI models. It is shown in Tables 7 and 8 that 3D TL models provided better approximations to 3D FSI models than 2D models. Considering the time cost and accuracy of the calculations, 3D TL modeling may be a better choice for clinical implementations.

\subsection{D structure-only models provided good approximation for FSI models}

While 3D FSI models may be more realistic, they take much more time to construct. Compared with 3D FSI models (used as the base for comparison), 3D structure-only models had modest errors (4.38\% and $1.78 \%$ for PWS and PWSn calculation). Structure-only models could provide PWS/PWSn calculations as good approximations to FSI models to save time. For flow simulation, 3D fluid-only models had larger error on Min-FSS calculation (11.29\% under maximum pressure condition). And the multi-patient study also showed significant differences among seven patients.

(3.59-36.63\%). FSI and flow-only model differences were greater for minimum FSS predictions, which is notable since low FSS is known to be related to plaque progression.

\subsection{Model comparisons and other factors affecting plaque mechanical conditions}

In addition to model assumptions, plaque morphology (especially cap thickness), component material properties and blood pressure all have large impact on model stress/ strain calculations. Figures 2 and 4 provide samples showing model solution differences were noticeably greater for plaques with thin cap and large lipid core. The current paper focused on model comparison and patient variations. Other comparisons using multi-patient data will be our future effort.

\subsection{Modeling limitations}

Patient-specific and tissue-specific material properties were not used in our study due to the difficulty in obtaining in vivo material data. Zero-stress conditions (opening angle) and multilayer morphology of vessels are also difficult to measure noninvasively in vivo. The resolution of IVUS images is still insufficient to accurately determine thin fibrous cap thickness, and higher resolution imaging approaches such as optical coherence tomography (OCT) should be adopted in the future to improve image segmentation accuracy.

\section{Conclusions}

Model differences had noticeable patient variations that must be considered when interpreting computational results from different models for different patients. Results from seven patients presented in this study showed that PWS and PWSn values would be overestimated by $2 \mathrm{D}$ models without shrink process. Cyclic bending process has an influence on the calculation of 3D FSI model, especially in locations with large curvature change. The effect of heart movement on coronary models should be considered. Considering time cost and accuracy of the stress and strain calculations, 3D TL models may be used for the mechanical analysis of atherosclerotic plaques and may be most practical for clinical implementations. FSI and flow-only model differences were greater for minimum FSS predictions, notable since low FSS is related to plaque progression. Structure-only models may provide 
PWS/PWSn calculations as reasonable approximations to FSI models to save time.

Acknowledgement This research was supported in part by National Sciences Foundation of China grants 11972117, 11672001, and a Jiangsu Province Science and Technology Agency grant BE2016785. Wang QY's research was supported in part by Postgraduate Research \& Practice Innovation Program of Jiangsu Province grant KYCX18_0156.

Open Access This article is licensed under a Creative Commons Attribution 4.0 International License, which permits use, sharing, adaptation, distribution and reproduction in any medium or format, as long as you give appropriate credit to the original author(s) and the source, provide a link to the Creative Commons licence, and indicate if changes were made. The images or other third party material in this article are included in the article's Creative Commons licence, unless indicated otherwise in a credit line to the material. If material is not included in the article's Creative Commons licence and your intended use is not permitted by statutory regulation or exceeds the permitted use, you will need to obtain permission directly from the copyright holder. To view a copy of this licence, visit http://creativecommons.org/licenses/by/4.0/.

\section{References}

Bathe KJ (2002) Theory and modeling guide, Vol I: ADINA; Vol II: ADINA-F, ADINA R \& D, Inc. Watertown, MA

Bluestein D, Alemu Y, Avrahami I, Gharib M, Dumont K, Ricotta JJ, Einav S (2008) Influence of microcalcifications on vulnerable plaque mechanics using FSI modeling. J Biomech 41:1111-1118. https://doi.org/10.1016/j.jbiomech.2007.11.029

Cardoso L, Weinbaum S (2014) Changing views of the biomechanics of vulnerable plaque rupture: a review. Ann Biomed Eng 42:415431. https://doi.org/10.1007/s10439-013-0855-x

Delfino A, Stergiopulos N, Moore JE, Meister JJ (1997) Residual strain effects on the stress field in a thick wall finite element model of the human carotid bifurcation. J Biomech 30:777-786. https://doi. org/10.1016/S0021-9290(97)00025-0

Friedman MH, Krams R, Chandran KB (2010) Flow interactions with cells and tissues: cardiovascular flows and fluid-structure interactions. Ann Biomed Eng 38:1178-1187. https://doi.org/10.1007/ s10439-010-9900-1

Gijsen FJ, Migliavacca F (2014) Plaque mechanics. J Biomech 47:763764. https://doi.org/10.1016/j.jbiomech.2014.01.031

Guo X, Zhu J, Maehara A, Monoly D, Samady H, Wang L, Billiar KL, Zheng J, Yang C, Mintz GS, Giddens DP, Tang D (2017) Quantify patient-specific coronary material property and its impact on stress/strain calculations using in vivo IVUS data and 3D FSI Models: a pilot study. Biomech Model Mechanobiol 16(1):333344. https://doi.org/10.1007/s10237-016-0820-3

Guo J, Wang L, Molony D, Samady H, Zheng J, Guo X, Maehara A, Mintz GS, Zhu J, Ma G, Tang D (2018) In Vivo intravascular ultrasound-based $3 \mathrm{~d}$ thin-walled model for human coronary plaque progression study transforming research to potential commercialization. Int J Comput Methods. https://doi.org/10.1142/ S0219876218420112

Holzapel GA, Sommer G, Auer M, Regitnig P, Ogden RW (2007) Layer-specific 3D residual deformations of human aortas with non-atherosclerotic intimal thickening. Ann Biomed Eng. 35:530 545. https://doi.org/10.1007/s10439-006-9252-z

Holzapfel GA, Stadler M, Schulze-Bause CA (2002) A layer-specific three dimensional model for the simulation of balloon angioplasty using magnetic resonance imaging and mechanical testing. Ann Biomed Eng 30:753-767. https://doi.org/10.1114/1.1492812
Holzapfel GA, Mulvihill JJ, Cunnane EM, Walsh MT (2014) Computational approaches for analyzing the mechanics of atherosclerotic plaques: a review. J Biomech 47:859-869. https://doi.org/ 10.1016/j.jbiomech.2014.01.011

Huang X, Yang C, Yuan C et al (2009) Patient-specific artery shrinkage and 3D zero-stress state in multi-component 3D FSI models for carotid atherosclerotic plaques based on in vivo MRI data. MCB Mol Cell Biomech 6:121-134

Huang Y, Teng Z, Sadat U, Graves MJ, Bennett MR, Gillard JH (2014) The influence of computational strategy on prediction of mechanical stress in carotid atherosclerotic plaques: comparison of 2D structure-only, 3D structure-only, one-way and fully coupled fluidstructure interaction analyses. J Biomech 47:1465-1471. https:// doi.org/10.1016/j.jbiomech.2014.01.030

Huang X, Yang C, Zheng J, Bach R, Muccigrosso D, Woodard PK, Tang D (2016) 3D MRI-based multicomponent thin layer structure only plaque models for atherosclerotic plaques. J Biomech 49:2726-2733. https://doi.org/10.1016/j.jbiomech.2016.06.002

Kural MH, Cai MC, Tang D, Gwyther T, Zheng J, Billiar KL (2012) Planar biaxial characterization of diseased human coronary and carotid arteries for computational modeling. J Biomech 45:790 798. https://doi.org/10.1016/j.jbiomech.2011.11.019

Li ZY, Howarth S, Trivedi RA, U-King-Im JM, Graves MJ, Brown A, Wang LQ, Gillard JH (2006) Stress analysis of carotid plaque rupture based on in vivo high resolution MRI. J Biomech 39:26112622. https://doi.org/10.1016/j.jbiomech.2005.08.022

Loree HM, Kamm RD, Stringfellow RG et al (1992) Effects of fibrous cap thickness on peak circumferential stress in model atherosclerotic vessels. Circ Res 71:850-858. https://doi.org/10.1161/01. RES.71.4.850

Mintz GS, Nissen SE, Anderson WD, Bailey SR, Erbel R, Fitzgerald PJ, Pinto FJ, Rosenfield K, Siegel RJ, Tuzcu EM, Yock PG (2001) American college of cardiology clinical expert consensus document on standards for acquisition, measurement and reporting of intravascular ultrasound studies (IVUS): a report of the American college of cardiology task force on clinical expert consensus documents. J Am Coll Cardiol 37:1478-1492. https:// doi.org/10.1016/S0735-1097(01)01175-5

Nair A, Kuban BD, Tuzcu EM, Schoenhagen P, Nissen SE, Vince DG (2002) Coronary plaque classification with intravascular ultrasound radiofrequency data analysis. Circulation 106:22002206. https://doi.org/10.1161/01.CIR.0000035654.18341

Ohayon J, Finet G, Treyve F et al (2005) A three-dimensional finite element analysis of stress distribution in a coronary atherosclerotic plaque: in-vivo prediction of plaque rupture location. Biomech Appl Comput Assist Surg 37:225-241

Ohayon J, Dubreuil O, Tracqui P, Le Floc'h S, Rioufol G, Chalabreysse L, Thivolet F, Pettigrew RI, Finet G (2007) Influence of residual stress/strain on the biomechanical stability of vulnerable coronary plaques: potential impact for evaluating the risk of plaque rupture. Am J Physiol 293:1987-1996. https://doi.org/ 10.1152/ajpheart.00018.2007

Pierce DM, Fastl TE, Rodriguez-Vila B, Verbrugghe P, Fourneau I, Maleux G, Herijgers P, Gomez EJ, Holzapfel GA (2015) A method for incorporating three-dimensional residual stretches/ stresses into patient-specific finite element simulation of arteries. J Mech Behav Biomed Mater 47:147-164. https://doi.org/ 10.1115/SBC2013-14232

Richardson PD, Davies MJ, Born GVR (1989) Influence of plaque configuration and stress distribution on fissuring of coronary atherosclerotic plaques. The Lancet 334:941-944. https://doi. org/10.1016/S0140-6736(89)90953-7

Samady H, Eshtehardi P, McDaniel MC, Suo J, Dhawan SS, Maynard C, Timmins LH, Quyyumi AA, Giddens DP (2011) Coronary artery wall shear stress is associated with progression and transformation of atherosclerotic plaque and arterial remodeling in 
patients with coronary artery disease. Circulation 124:779-788. https://doi.org/10.1161/CIRCULATIONAHA.111.021824

Speelman L, Bosboom EM, Schurink GW, Buth J, Breeuwer M, Jacobs MJ, van de Vosse FN (2009) Initial stress and nonlinear material behavior in patient-specific AAA wall stress analysis. J Biomech 42:1713-1719. https://doi.org/10.1016/j.jbiomech. 2009.04.020

Stone PH, Saito S, Takahashi S et al (2012) Prediction of progression of coronary artery disease and clinical outcomes using vascular profiling of endothelial shear stress and arterial plaque characteristics: the PREDICTION Study. Circulation 126:172-181. https:// doi.org/10.1161/CIRCULATIONAHA.112.096438

Tang D, Yang C, Zheng J, Woodard PK, Sicard GA, Saffitz JE, Yuan C (2004) 3D MRI-based multi-component FSI models for atherosclerotic plaques a 3-D FSI model. Ann Biomed Eng 32:947-960. https://doi.org/10.1023/B:ABME.0000032457.10191.e0

Tang D, Kamm RD, Yang C et al (2014) Image-based modeling for better understanding and assessment of atherosclerotic plaque progression and vulnerability: data, modeling, validation, uncertainty and predictions. J Biomech 47:834-846. https://doi.org/10.1016/j. jbiomech.2014.01.012

Teng Z, Canton G, Yuan C, Ferguson M, Yang C, Huang X, Zheng J, Woodard PK, Tang D (2010) 3D critical plaque wall stress is a better predictor of carotid plaque rupture sites than flow shear stress: an in vivo MRI-based 3D FSI study. J Biomech Eng 132:031007. https://doi.org/10.1115/1.4001028

Wang H, Zheng J, Wang L, Maehara A, Yang C, Muccigrosso D, Bach R, Mintz GS, Tang D (2015a) Using 2D In Vivo IVUS-based models for human coronary plaque progression analysis and comparison with $3 \mathrm{~d}$ fluid-structure interaction models a multi-patient study. MCB Mol Cell Biomech 12:107-122. https://doi.org/10. 1016/j.proeng.2015.11

Wang L, Zheng J, Maehara A, Yang C, Billiar KL, Wu Z et al (2015b) Morphological and stress vulnerability indices for human coronary plaques and their correlations with cap thickness and lipid percent an ivus-based fluid-structure interaction multi-patient study. PLoS Comput Biol. https://doi.org/10.1371/journal.pcbi. 1004652

Wang L, Zhu J, Samady H et al (2017a) Effects of residual stress, axial stretch, and circumferential shrinkage on coronary plaque stress and strain calculations: a modeling study using ivus-based nearidealized geometries. J Biomech Eng. https://doi.org/10.1115/1. 4034867

Wang Q, Canton G, Guo J, Guo X, Hatsukami TS, Billiar KL, Yuan C, Wu ZY, Tang D (2017b) MRI-based patient-specific human carotid atherosclerotic vessel material property variations in patients vessel location and long-term follow up. PLoS ONE. https://doi.org/10.1371/journal.pone.0180829

Yang C, Bach RG, Zheng J et al (2009) In vivo IVUS-based 3-D fluidstructure interaction models with cyclic bending and anisotropic vessel properties for human atherosclerotic coronary plaque mechanical analysis. IEEE Trans Biomed Eng 56(10):2420-2428. https://doi.org/10.1109/TBME.2009.2025658

Publisher's Note Springer Nature remains neutral with regard to jurisdictional claims in published maps and institutional affiliations. 